Original article

\title{
Serum Uric Acid and Cardiovascular Risk Among Portuguese Adolescents
}

\author{
Jean-Pierre Gonçalves, M.D. ${ }^{\text {a,b,c }}$, Elisabete Ramos, Ph.D. ${ }^{\text {a,b,c }}$, Milton Severo, Ph.D. ${ }^{\text {a,b,c }}$, \\ Max C. Y. Wong, Ph.D. d, Ken K. Ong, Ph.D. e , David B. Dunger, Ph.D. d, and Carla Lopes, Ph.D. ${ }^{\text {a,b,c,* }}$ \\ ${ }^{a}$ Department of Epidemiology, Institute of Public Health, University of Porto, Porto, Portugal \\ ${ }^{\mathrm{b}}$ Department of Clinical Epidemiology, Predictive Medicine and Public Health, University of Porto Medical School, Porto, Portugal \\ ${ }^{\mathrm{c}}$ Cardiovascular Research and Development Unit, University of Porto Medical School, Porto, Portugal \\ ${ }^{\mathrm{d}}$ Department of Pediatrics, University of Cambridge, Cambridge, United Kingdom \\ ${ }^{\mathrm{e}}$ Institute of Metabolic Science, Medical Research Council Epidemiology Unit, Cambridge, United Kingdom
}

Article history: Received March 20, 2014; Accepted November 12, 2014

Keywords: Classes; Cardiovascular risk; Serum uric acid

\section{See Related Editorial p. 363}

\section{A B S T R A C T}

Purpose: The aim of the study was to investigate the association between serum uric acid (SUA) and cardiovascular risk classes (CRCs) in adolescents using a cluster-based approach.

Methods: A cross-sectional evaluation was carried out in the 2007-2008 school year, including adolescents born in 1990 and enrolled in the schools of Porto, Portugal. The analysis included 1,286 adolescents. To identify CRC, a normal mixture model was performed including several biological cardiovascular risk factors. A multinomial logistic regression model was applied to explore the association between SUA and each CRC.

Results: Three classes were extracted using model-based cluster analysis (low, medium, and high CRC). The high CRC accounted for the smallest proportion of participants (5.6\%) and represented the adolescents with higher waist circumference, systolic and diastolic blood pressures, total cholesterol, triglycerides, and insulin levels. Adolescents at increased risk of cardiovascular disease had significantly higher mean concentrations of SUA compared with adolescents at low cardiovascular risk (55.0 vs. $51.5 \mathrm{mg} / \mathrm{L}$ in males and 41.9 vs. $37.6 \mathrm{mg} / \mathrm{L}$ in females). After adjustment and considering low CRC as reference, SUA was positively associated with high CRC in both sexes (odds ratio, 1.04 ; $95 \%$ confidence interval, $1.00-1.07$ in males; and odds ratio, $1.04 ; 95 \%$ confidence interval, 1.01-1.07 in females).

Conclusions: Among 17-year-old adolescents, SUA increases were positively associated with higher CRC.

\section{IMPLICATIONS AND CONTRIBUTION}

Previous studies have shown strong associations between serum uric acid and cardiovascular diseases in adults. Serum uric acid was positively associated with high cardiovascular risk class in adolescents. To our knowledge, the association of SUA and cardiovascular risk factor clustering, using data-driven approach, has not been evaluated in prior studies.

(C) 2015 Society for Adolescent Health and Medicine. All rights reserved.

Conflicts of Interest: The authors have no conflicts of interest or financial disclosures to disclose.

* Address correspondence to: Carla Lopes, Ph.D., Department of Clinical Epidemiology, Predictive Medicine and Public Health and Cardiovascular Research and Development Unit, University of Porto Medical School, Alameda Professor Hernâni Monteiro, Porto 4200-319, Portugal.

E-mail address: carlal@med.up.pt (C. Lopes).
Adolescence is a generally healthy period when important behavioral patterns are developed and preventable biological risk factors such as high blood pressure, high blood cholesterol, and overweight underlie cardiovascular disease (CVD) and other chronic diseases [1].

Serum uric acid (SUA) is the end product of the metabolism of purine compounds. Previous studies have suggested that 
SUA stimulates vascular smooth muscle proliferation and is also associated with deleterious effects on endothelial function, platelet adhesion and aggregation, and oxidative metabolism $[2,3]$.

Elevated SUA has frequently been described as a correlate of the development and progression of CVD. Several cohort studies have shown positive and significant associations between SUA and CVDs in adult population [4,5]. Systematic reviews and meta-analyses have also confirmed this association [6,7].

Although CVD generally occurs in adulthood, the process of atherosclerosis can begin in childhood [8]. Previous crosssectional data have shown a close relationship between SUA concentrations and cardiovascular (CV) risk factors in children and adolescents [9]. Findings from a cohort study of adolescents from Taiwan showed that adolescents with hyperuricemia have an increased risk of mortality, especially because of kidney and CVDs [10]. However, the specific role of SUA in the development of CVD remains unclear among adults and even more so among children and adolescents. In a young population, mostly free of chronic disorders, it would be helpful to use data-driven approach to better define how CV risk factors tend to cluster in adolescence and their association with SUA.

The aim of the study was to investigate the association between SUA and CV risk in adolescents, using a cluster-based approach to identify those at high CV risk.

\section{Methods}

Subjects

This study was developed as part of the EPITeen study, a cohort of adolescents born in 1990 and attending public and private schools in Porto, Portugal [11]. Recruitment of the cohort took place during the 2003-2004 school year when adolescents were 13 years old, and 2,160 (77.7\% from public schools and $76.7 \%$ from private schools, $p=.710$ ) agreed to participate by providing information for at least part of the planned protocol.

During the 2007-2008 school year, the first follow-up was performed using the same protocol and 1,716 (79.4\%) of the participants were reevaluated. Additionally, 783 adolescents who were born in 1990 but did not attend school in the Porto area at baseline were evaluated for the first time.

A cross-sectional analysis was carried out including 1,286 17year-old adolescents with complete information on anthropometrics and blood samples.

\section{Data collection}

The evaluation included self-administered questionnaires, comprising information on social, demographic, and behavioral characteristics and individual and family history of CVD.

Sports activity was considered as practicing some sports, outside the compulsory school curriculum, independently of the frequency or intensity.

Adolescents were classified regarding their use of alcohol and tobacco as never users if they have never drunk alcoholic drinks and if they have never smoked. If they reported only experimented those substances, either occasionally or regularly, they were classified as ever drinkers or smokers, respectively.

A physical examination was also performed at school, by a team of experienced nurses, nutritionists, and physicians. Anthropometrics was obtained with the subjects in light indoor clothes and no shoes. Weight was measured using a Tanita bioimpedance scale (Tanita TBF-300; Tanita Corporation of America, Inc., Arlington Heights, IL) with subjects positioned in the center of the weighing platform so that their weight was evenly distributed. Height was measured with a portable stadiometer, with subjects standing with their heels together and their head positioned in the Frankfort horizontal plane, with heels, buttocks, shoulder blades, and head against the back of the stadiometer. Body mass index (BMI) was calculated as weight in kilograms divided by the square of height in meters $\left(\mathrm{kg} / \mathrm{m}^{2}\right)$.

Waist circumference (WC) was measured to the nearest centimeter, midway between the lower limit of the rib cage and the iliac crest, with the subject standing, using a flexible and nondistensible tape, and avoiding pressure on the tissues [12].

Blood pressure was measured with a mercury sphygmomanometer using the auscultatory method, following the recommendations of the American Academy of Pediatrics [13]. We considered the mean of two readings taken on a single occasion, separated by at least 10 minutes of rest, with a third reading being taken if the difference between the first two readings was greater than $5 \mathrm{~mm} \mathrm{Hg}$.

A 12-hour overnight fasting blood sample was drawn from consenting participants. Blood was centrifuged, serum and plasma were divided into aliquots, and immediately analyzed or stored frozen at $-80^{\circ} \mathrm{C}$ until used. SUA, glucose, total cholesterol, high-density lipoprotein cholesterol (HDLc), and triglycerides were measured using standard automatic routine enzymatic methods in use at the central pathology laboratory of the University Hospital of São João, Porto.

The homeostasis model assessment (HOMA) was used to calculate indices of insulin resistance and insulin secretion for each patient. The HOMA Calculator v2.2.2 (Diabetes Trials Unit, University of Oxford, Oxford, UK) used fasting glucose and insulin to generate the index of insulin resistance (HOMA2-IR) [14].

\section{Ethical considerations}

Written informed consent was obtained from adolescents and legal guardians. The study protocol complied with the Declaration of Helsinki and was approved by the Ethics Committee of the University Hospital of São João in Porto, Portugal.

\section{Statistical analysis}

Quantitative continuous variables according to sex-specific SUA quartiles and CRCs were compared using one-way analysis of variance. Categorical variables were compared using the chi-square test.

Pearson correlation analysis was used to examine the relationship between biological CV risk variables (systolic blood pressure [SBP], diastolic blood pressure [DBP], BMI, WC, HDLc, total cholesterol, triglycerides, glucose, insulin, and HOMA2-IR). For variables highly correlated (Pearson coefficient $\geq .800$ ), only one was included in the cluster analysis performed to identify CRCs [15].

Model-based clustering is based on the assumption that the observed data come from a population consisting of several subpopulations. Using this analysis, cluster solutions are not affected by changes in a variable's unit of measurement when dealing with normal distributions with unknown variances. For this reason, we did not standardize the variables by each standard deviation unit.

To the cluster analyses, the number of classes was defined according to the Bayesian information criterion [16]. 
We applied a multinomial logistic regression model to explore the association between SUA and each CRC. Statistical testing of the linearity assumption was performed.

Statistical analysis was performed using the $\mathrm{R}$ Statistical Software (version 2.7.1, R Foundation for Statistical Computing, Austria).

\section{Results}

Comparisons between adolescents with and without followup revealed only differences for alcohol consumption and sports practice. Final sample participants practice sports more often and have lower proportion of current drinkers in female adolescents. For smoking, age at menarche, WC, SBP, and DBP, there were no differences between groups.

Characteristics of the participants according to sex-specific SUA quartiles are summarized in Table 1. BMI, WC, insulin, and
HOMA2-IR means tended to increase with increasing quartiles of SUA concentrations in both males and females. This trend was also observed for triglycerides and total cholesterol in males. HDLc mean decreased with increasing categories of SUA in males.

The correlations between the biological CV risk factors analyzed in this study were performed to decide the final variables to include in the cluster models. As expected, a strong correlation between insulin and HOMA2-IR (Pearson coefficient: $.999, p<.001$ ) and between WC and BMI (Pearson coefficient: $.844, p<.001$ ) was observed, and for that reason only, WC and insulin were considered in the final model-based clustering. For all the other variables, correlation coefficients were lower than .5.

Considering data on SBP, DBP, WC, HDLc, total cholesterol, triglycerides, glucose, and insulin, three CRCs were extracted using model-based cluster analysis.

Table 2 provides the description of the three CRCs (low, medium, and high). The low CV risk first accounted for the largest

Table 1

Descriptive analysis of EPITeen cohort adolescents aged 17 years according to sex-specific serum uric acid (SUA) quartiles, by sex (Porto, Portugal 2007-2008)

\begin{tabular}{|c|c|c|c|c|c|}
\hline & \multicolumn{4}{|c|}{$\mathrm{SUA}, \mathrm{mg} / \mathrm{L}(\mathrm{N}=1,286)$} & \multirow[t]{2}{*}{$p$ value } \\
\hline & $\mathrm{Q} 1, \mathrm{n}=325$ & $\mathrm{Q} 2, \mathrm{n}=322$ & $\mathrm{Q} 3, \mathrm{n}=317$ & $\mathrm{Q} 4, \mathrm{n}=322$ & \\
\hline Female & $\leq 32.8$ & $>32.8-\leq 37.7$ & $>37.7-\leq 43.2$ & $>43.2$ & \\
\hline Male & $\leq 46.2$ & $>46.2-\leq 52.2$ & $>52.2-\leq 58.0$ & $>58.0$ & \\
\hline \multicolumn{6}{|c|}{ Smoking (ever smokers); n (\%) } \\
\hline 우 & $75(43.4)$ & $78(46.2)$ & $70(42.2)$ & $79(46.5)$ & .823 \\
\hline 0 & $62(41.1)$ & $66(44.3)$ & $59(40.1)$ & $60(40.5)$ & .883 \\
\hline \multicolumn{6}{|c|}{ Alcohol (ever drinkers); n (\%) } \\
\hline q & $139(80.3)$ & $132(78.1)$ & $138(84.7)$ & $137(81.1)$ & .496 \\
\hline$\hat{0}$ & $127(84.7)$ & $132(89.3)$ & $126(85.7)$ & $127(84.7)$ & .528 \\
\hline \multicolumn{6}{|c|}{ Sports practice (yes); n (\%) } \\
\hline q & $70(41.9)$ & $75(45.7)$ & $80(49.1)$ & $67(40.6)$ & .401 \\
\hline 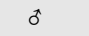 & $104(71.2)$ & $109(74.1)$ & $104(72.2)$ & $109(75.2)$ & .870 \\
\hline \multicolumn{6}{|c|}{ Age at menarche (years); mean (SD) } \\
\hline 운 & $12.31(1.19)$ & $12.36(1.42)$ & $12.19(1.29)$ & $12.34(1.38)$ & .622 \\
\hline o & - & - & - & - & - \\
\hline \multicolumn{6}{|c|}{ Body mass index $\left(\mathrm{kg} / \mathrm{m}^{2}\right)$; mean (SD) } \\
\hline q & $21.92(2.89)$ & $21.61(2.62)$ & $22.34(3.01)$ & $23.51(4.36)$ & .001 \\
\hline 0 & $21.69(3.00)$ & $21.96(3.01)$ & $22.50(3.10)$ & $24.32(3.85)$ & .001 \\
\hline \multicolumn{6}{|c|}{ Waist circumference $(\mathrm{cm})$; mean (SD) } \\
\hline q & $74.06(7.53)$ & $73.67(7.36)$ & $74.77(7.45)$ & $77.24(10.09)$ & .001 \\
\hline 0 & $76.01(7.24)$ & $77.09(7.74)$ & $78.64(7.64)$ & $\begin{array}{l}82.72(10 . \\
32)\end{array}$ & .001 \\
\hline \multicolumn{6}{|c|}{ Triglycerides (g/L); mean (SD) } \\
\hline o & $.70(.30)$ & $.74(.33)$ & $.76(.37)$ & $.73(.32)$ & .491 \\
\hline 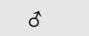 & $.64(.24)$ & $.65(.31)$ & $.66(.28)$ & $.73(.35)$ & .045 \\
\hline \multicolumn{6}{|c|}{ Total cholesterol $(\mathrm{g} / \mathrm{L})$; mean $(\mathrm{SD})$} \\
\hline 우 & $1.68(.31)$ & $1.70(.29)$ & $1.71(.33)$ & $1.72(.36)$ & .710 \\
\hline 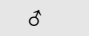 & $1.46(.25)$ & $1.47(.27)$ & $1.55(.28)$ & $1.52(.27)$ & .011 \\
\hline \multicolumn{6}{|c|}{ HDLc $(g / L) ;$ mean $(S D)$} \\
\hline 우 & $.60(.12)$ & $.60(.12)$ & $.59(.11)$ & $.57(.13)$ & .059 \\
\hline o & $.52(.10)$ & $.52(.11)$ & $.51(.10)$ & $.47(.09)$ & .001 \\
\hline \multicolumn{6}{|c|}{$\mathrm{SBP}(\mathrm{mm} \mathrm{Hg}) ;$ mean (SD) } \\
\hline q & $112.56(10.16)$ & $112.59(10.82)$ & $112.92(12.81)$ & 111.75 (10.39) & .794 \\
\hline 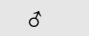 & $119.86(12.87)$ & $119.26(13.01)$ & $118.56(11.48)$ & $120.40(12.49)$ & .610 \\
\hline \multicolumn{6}{|c|}{$\mathrm{DBP}(\mathrm{mm} \mathrm{Hg}) ;$ mean $(\mathrm{SD})$} \\
\hline 임 & $67.76(8.42)$ & $66.14(8.64)$ & $66.96(8.99)$ & $67.22(8.15)$ & .361 \\
\hline$\widehat{0}$ & $70.00(9.63)$ & $68.77(9.43)$ & $70.83(9.67)$ & $70.40(9.63)$ & .277 \\
\hline \multicolumn{6}{|c|}{ Glucose $(\mathrm{g} / \mathrm{L})$; mean $(\mathrm{SD})$} \\
\hline 우 & $.83(.07)$ & $.84(.08)$ & $.82(.08)$ & $.83(.07)$ & .452 \\
\hline 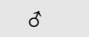 & $.87(.08)$ & $.87(.15)$ & $.86(.08)$ & $.88(.08)$ & .621 \\
\hline \multicolumn{6}{|c|}{ Insulin $(\mu \mathrm{IU} / \mathrm{mL})$; mean $(\mathrm{SD})$} \\
\hline q & $6.00(3.56)$ & 5.39 (3.59) & $5.48(3.83)$ & $6.51(4.89)$ & .038 \\
\hline 0 & $5.29(3.23)$ & $4.95(3.08)$ & $6.15(5.25)$ & $6.36(4.65)$ & .008 \\
\hline \multicolumn{6}{|c|}{ HOMA2-IR; mean (SD) } \\
\hline 우 & $.76(.45)$ & $.69(.45)$ & $.69(.48)$ & $.83(.60)$ & .036 \\
\hline 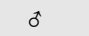 & $.69(.41)$ & $.64(.40)$ & $.79(.66)$ & $.82(.57)$ & .009 \\
\hline
\end{tabular}

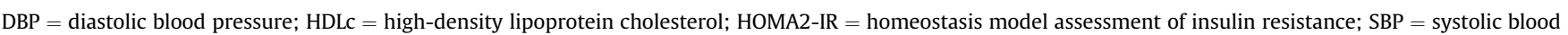
pressure; SD = standard deviation. 
Table 2

Characterization of classes with low, medium, and high cardiovascular (CV) risk identified in the EPITeen cohort adolescents aged 17 years, by sex (Porto, Portugal 2007-2008)

\begin{tabular}{|c|c|c|c|c|}
\hline & \multicolumn{3}{|c|}{$\mathrm{CV}$ risk classes $(\mathrm{N}=1,286)$} & \multirow[t]{2}{*}{$p$ value } \\
\hline & Low $(\mathrm{n}=769)$ & Medium $(\mathrm{n}=445)$ & High $(n=72)$ & \\
\hline Female & 383 & 270 & 29 & \\
\hline Male & 386 & 175 & 43 & \\
\hline \multicolumn{5}{|c|}{ Waist circumference (cm); mean (SD) } \\
\hline 우 & $72.86(6.12)$ & $76.8(9.47)$ & $84.7(10.30)$ & .001 \\
\hline 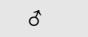 & $75.78(5.57)$ & $82.00(9.99)$ & $90.33(11.18)$ & .001 \\
\hline \multicolumn{5}{|c|}{ Triglycerides $(\mathrm{g} / \mathrm{L})$; mean $(\mathrm{SD})$} \\
\hline q & $.57(.13)$ & $.89(.31)$ & $1.38(.67)$ & .001 \\
\hline 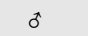 & $.54(1.34)$ & $.85(.28)$ & $1.09(.57)$ & .001 \\
\hline \multicolumn{5}{|c|}{ Total cholesterol (g/L); mean (SD) } \\
\hline q & $1.63(.26)$ & $1.77(.33)$ & $2.07(.50)$ & .001 \\
\hline 0 & $1.45(.22)$ & $1.55(.30)$ & $1.77(.34)$ & .001 \\
\hline \multicolumn{5}{|c|}{ HDLc $(g / L)$; mean $(S D)$} \\
\hline 운 & $.59(.11)$ & $.59(.13)$ & $.59(.13)$ & .479 \\
\hline 0 & $.52(.10)$ & $.47(.11)$ & $.49(.10)$ & .001 \\
\hline \multicolumn{5}{|c|}{ SBP (mm Hg); mean (SD) } \\
\hline ஒ & $111.62(10.94)$ & $112.99(9.90)$ & $118.45(18.83)$ & .003 \\
\hline 0 & $118.79(11.94)$ & $118.46(10.79)$ & $130.44(17.65)$ & .001 \\
\hline \multicolumn{5}{|c|}{ DBP (mm Hg); mean (SD) } \\
\hline 우 & $66.60(8.13)$ & $67.02(8.35)$ & $72.69(13.10)$ & .001 \\
\hline $0^{*}$ & $60.30(8.77)$ & $69.93(8.26)$ & 76.56 (16.89) & .001 \\
\hline \multicolumn{5}{|c|}{ Glucose (g/L); mean (SD) } \\
\hline q & $.83(.08)$ & $.83(.07)$ & $.82(.08)$ & .454 \\
\hline$\delta$ & $.86(.08)$ & $.88(.07)$ & $.93(.26)$ & .001 \\
\hline \multicolumn{5}{|c|}{ Insulin $(\mu \mathrm{IU} / \mathrm{mL}) ;$ mean $(\mathrm{SD})$} \\
\hline q & $4.16(2.14)$ & $7.26(3.49)$ & $15.00(8.27)$ & .001 \\
\hline$\sigma^{+}$ & $4.25(2.09)$ & $7.26(3.50)$ & $12.13(9.44)$ & .001 \\
\hline
\end{tabular}

$\mathrm{DBP}=$ diastolic blood pressure; HDLc = high-density lipoprotein cholesterol; $\mathrm{SBP}=$ systolic blood pressure; $\mathrm{SD}=$ standard deviation

proportion of the total participants (59.8\%) and represents the adolescents (males and females) with lower SBP, DBP, WC, cholesterol, triglycerides, and insulin levels. Adolescent males in this subgroup also had lower glucose and higher HDLc levels. On the other hand, the high CRC accounted for the smallest proportion of participants (5.6\%) and represents the adolescents with higher SBP, DBP, WC, cholesterol, triglycerides, and insulin levels. Adolescent males in this subgroup had significantly higher glucose levels. Increasing the CRC, it is notorious that the biological variables' means also increases (except for HDL). No significant differences were found between CRCs regarding smoking, alcohol, and physical activity (data not shown).

The SUA values ranged from 12.1 to $86.4 \mathrm{mg} / \mathrm{L}$ ( $₫$ : $13.2-86.4$; : $12.1-68.5$ ), with a mean of $52.6 \mathrm{mg} / \mathrm{L}(9.9)$ in males and $38.2 \mathrm{mg} / \mathrm{L}$ (8.2) in females. The SUA mean values according to CRC by sex are presented graphically in Figure 1. In both genders, adolescents at increased risk of CVD had higher mean values of SUA.

Considering low CRC as reference, there is a significant positive relationship between SUA and medium and high CRCs in both sexes (Table 3). The relationship between SUA and CRCs is linear. In univariated analysis, the odds ratio for the association between SUA and high CRC was 1.04 (95\% confidence interval, 1.00-1.07) in males and 1.06 (95\% confidence interval, 1.02-1.11) in females. After adjustment, each SUA ( $\mathrm{mg} / \mathrm{L})$ unit increase was associated with a $4 \%$ increased odds of high CV risk, in both sexes.

\section{Discussion}

The present study showed that SUA is positively associated with high CRC among adolescents.

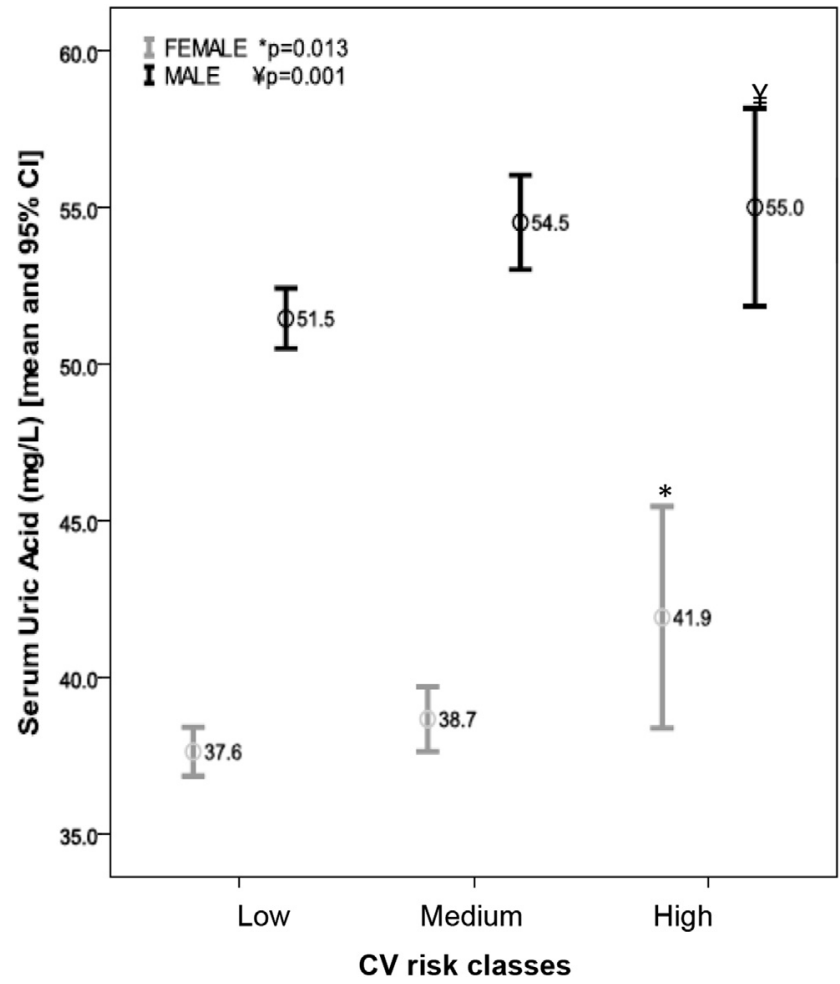

Figure 1. Mean and 95\% confidence interval (CI) of serum uric acid (mg/L) according to cardiovascular (CV) risk classes by sex.

Previous cross-sectional data have shown a close relationship between SUA concentrations and individual CV risk factors in children and adolescents $[9,17]$. A study in the U.S. children and adolescents shows that concentrations of SUA were significantly associated with abdominal obesity, hypertriglyceridemia, low HDL, high blood pressure, and hyperglycemia. The strongest association was between SUA and abdominal obesity [9].

Detection of high-risk individuals is reliable by CVD risk clusters approach because CV risk factors tend to cluster and work synergistically $[1,18]$. As WC is widely used as a proxy measure of visceral adiposity, it was included in cluster-based model instead of BMI. Insulin and HOMA have been used as IR

Table 3

Association between serum uric acid $(\mathrm{mg} / \mathrm{L})$ and cardiovascular $(\mathrm{CV})$ risk classes, by $\operatorname{sex}(\mathrm{N}=1,286)$, based on multinomial logistic regression analysis

\begin{tabular}{|c|c|c|c|}
\hline & \multicolumn{3}{|c|}{ CV risk classes } \\
\hline & Low & Medium & High \\
\hline & OR (95\% CI) & OR (95\% CI) & OR (95\% CI) \\
\hline \multicolumn{4}{|l|}{ Male } \\
\hline Crude model & 1 & $1.03(1.01-1.05)$ & $1.04(1.00-1.07)$ \\
\hline Adjusted model $^{\mathrm{a}}$ & 1 & $1.03(1.01-1.05)$ & $1.04(1.00-1.07)$ \\
\hline \multicolumn{4}{|l|}{ Female } \\
\hline Crude model & 1 & $1.02(1.00-1.04)$ & $1.06(1.02-1.11)$ \\
\hline Adjusted model $^{\mathrm{b}}$ & 1 & $1.03(1.01-1.05)$ & $1.04(1.01-1.07)$ \\
\hline
\end{tabular}

Low CV risk class is the reference category.

$\mathrm{CI}=$ confidence interval; $\mathrm{OR}=$ odds ratio.

a Adjusted for smoking (current smoker), alcohol (current drinker), and sports practice (yes/no)

b Adjusted for smoking (current smoker), alcohol (current drinker), sports practice (yes/no), and age at menarche (years). 
proxy measures. Similar correlation of WC with insulin and HOMA has been reported in pediatric studies [19]. Considering that fasting insulin is a biological variable mandatory to generate HOMA and both were highly correlated, the inclusion of insulin in cluster analysis was an obvious option [20].

Clustering of $\mathrm{CV}$ risk factors have been previously documented among adolescents [21,22]. To our knowledge, the association of SUA and CV risk factors clustering, using data-driven approach, has not been evaluated in prior studies.

Model-based cluster analysis seems to be a valuable and adequate analytic tool for research in CV diseases. It can be used to explore heterogeneous population to better integrate distinctive processes into a consistent conceptual framework and also as an alternative method for comparing groups when the assumption of measurement invariance is either untenable or unreasonable across different populations.

Using a model-based cluster analysis, three classes were extracted (low, medium, and high CRCs). The participant distribution for each class is based on similarity attending eight different physiological $\mathrm{CV}$ risks. The descriptive analysis revealed higher biological CV risk factors and lower HDLc means for the high CV class. A total of 73 adolescents (5.6\%) were grouped on the highest CRC (class 3 ). This class probably represents the adolescents with metabolic syndrome (MS). In the present sample, using the de Ferranti criteria [23], the MS prevalence was 6.3\% (data not shown). Similar prevalence for adolescents was described in Brazilian and North American populations [24,25]. A systematic review of literature brought evidence that the prevalence of MS among adolescents varied from 2.2\% to $52.1 \%$ depending on the criterion adopted and the studied population [26]. The variation of MS prevalence among studies is essentially explained by the existence of several adolescent definitions [27,28].

In the present study, high CRC seems to represent mainly the adolescents with adiposity and insulin resistance. Some studies showed that SUA level is independently associated with serum leptin level and suggested that leptin could be a factor responsible for hyperuricemia in obese patients [29] and with insulin resistance syndrome [30]. In a study of a 14-year-old population, high adiposity was the strongest independent predictor of inflammatory markers, accounting for $28 \%$ of variance in uric acid [31]. There is evidence of a common pathway underlying insulin resistance, obesity, and lipid production. Obesity may be linked to decreased glucose tolerance and hyperinsulinemia [32]. Adipocytes can increase the production of free fatty acids and slow down glucose uptake stimulating hepatic glucose production and suppressing pancreatic insulin secretion [33]. Moreover, increased lipid production by the liver is associated with an overall increase in total glucose production, additional stress on beta cell function, and insulin production [34].

Gender differences in SUA concentrations were observed. Females had lower SUA mean in all CRCs. Other studies reported gender differences in the normal SUA levels [35,36]. This difference becomes most obvious during adolescence and has been attributed to the effect of estrogen. However, the magnitude of association between SUA and high CV risk did not differ by sex.

This study has several strengths and limitations. Its strengths include the use of large, population-based sample from a 17year-old adolescents cohort enrolled in public and private schools. Participants with missing information or who were lost to follow-up were excluded. Comparisons between adolescents with and without follow-up revealed only differences for alcohol consumption and sports practice. Final sample is more physically active with lower proportion of female drinkers indicating a possible selection of healthier participants. Hypothesizing a possible interference between SUA and CV classes associations, it is certainly an underestimation and not overestimation of the real association.

A limitation of the study was the relatively small sample size in the high CV risk group. Even so, a significant positive association between SUA and high CRC was found. The errors attributed to self-report information in school-based adolescent health surveys, even in those anonymous, are usually mentioned as a possible limitation because they could lead to an underestimation of exposure and outcome. However, in the present study, the main exposure and outcome were objectively assessed, which constitutes the strength of the study. Moreover, data were collected by trained interviewers. The cross-sectional nature of our data does not allow us to discern the causal relationships. So, we are unable to identify if SUA increase leads to higher CRC or if it occurs in the opposite way. Further longitudinal studies would be useful to clarify the independent role of high SUA in CV adolescent's health and how these various risk factors interact over time.

The present study described that SUA was positively associated with higher CRC, in a population cohort of 17-year-old adolescents. The study supports that the relation between SUA levels and CV risk factors clustering appears to be established early in the life course.

\section{Funding Sources}

The study was supported by a grant from the Portuguese Foundation for Science and Technology: FCOMP-01-0124-FEDER015750 (Ref. FCT PTDC/SAU-EPI/115254/2009).

\section{References}

[1] Rodgers A, Ezzati M, Vander Hoorn S, et al. Distribution of major health risks: Findings from the Global Burden of Disease study. PLoS Med 2004;1: e27.

[2] Johnson RJ, Kang DH, Feig D, et al. Is there a pathogenetic role for uric acid in hypertension and cardiovascular and renal disease? Hypertension 2003; 41:1183-90.

[3] Kanellis J, Kang DH. Uric acid as a mediator of endothelial dysfunction, inflammation, and vascular disease. Semin Nephrol 2005;25:39-42.

[4] Kivity S, Kopel E, Maor E, et al. Association of serum uric acid and cardiovascular disease in healthy adults. Am J Cardiol 2013;111:1146-51.

[5] Goncalves JP, Oliveira A, Severo M, et al. Cross-sectional and longitudinal associations between serum uric acid and metabolic syndrome. Endocrine 2012;41:450-7.

[6] Kim SY, Guevara JP, Kim KM, et al. Hyperuricemia and coronary heart disease: A systematic review and meta-analysis. Arthritis Care Res (Hoboken) 2010;62:170-80.

[7] Huang H, Huang B, Li Y, et al. Uric acid and risk of heart failure: A systematic review and meta-analysis. Eur J Heart Fail 2014;16:15-24.

[8] Expert panel on integrated guidelines for cardiovascular health and risk reduction in children and adolescents: Summary report. Pediatrics 2011; 128(Suppl 5):S213-56.

[9] Ford ES, Li C, Cook S, Choi HK. Serum concentrations of uric acid and the metabolic syndrome among U.S. children and adolescents. Circulation 2007; 115:2526-32.

[10] Hsia SH, Chou IJ, Kuo CF, et al. Survival impact of serum uric acid levels in children and adolescents. Rheumatol Int 2013;33:2797-802.

[11] Ramos E, Barros H. Family and school determinants of overweight in 13-year-old Portuguese adolescents. Acta Paediatr 2007;96:281-6.

[12] Rotwein P, Pollock KM, Watson M, Milbrandt JD. Insulin-like growth factor gene expression during rat embryonic development. Endocrinology 1987; $121: 2141-4$.

[13] The fourth report on the diagnosis, evaluation, and treatment of high blood pressure in children and adolescents. Pediatrics 2004;114(2 Suppl 4th Report):555-76. 
[14] Shizume K. [Development of recombinant human growth hormone and its clinical use]. Nihon Rinsho Jpn J Clin Med 1989;47:2391-4.

[15] Fraley C, Raftery AE. Enhanced software for model-based clustering, discriminant analysis, and density estimation: MCLUST. J Classif 2003:20:263-86.

[16] Fraley C, Raftery AE. Model-based clustering, discriminant analysis, and density estimation. J Am Stat Assoc 2002;97:611-31.

[17] Denzer C, Muche R, Mayer H, et al. Serum uric acid levels in obese children and adolescents: Linkage to testosterone levels and pre-metabolic syndrome. J Pediatr Endocrinol Metab 2003;16:1225-32.

[18] Kemper HC, Post GB, Twisk JW, van Mechelen W. Lifestyle and obesity in adolescence and young adulthood: Results from the Amsterdam Growth and Health Longitudinal Study (AGAHLS). Int J Obes Relat Metab Disord 1999;23(Suppl 3):S34-40.

[19] Kondaki K, Grammatikaki E, Pavon DJ, et al. Comparison of severa anthropometric indices with insulin resistance proxy measures among European adolescents: The Helena Study. Eur J Pediatr 2011;170:731-9.

[20] Laakso M. How good a marker is insulin level for insulin resistance? Am Epidemiol 1993;137:959-65.

[21] Grundy SM. Obesity, metabolic syndrome, and cardiovascular disease J Clin Endocrinol Metab 2004;89:2595-600.

[22] Berenson GS, Srinivasan SR, Bao W, et al. Association between multiple cardiovascular risk factors and atherosclerosis in children and young adults. The Bogalusa Heart Study. N Engl J Med 1998;338:1650-6.

[23] de Ferranti SD, Gauvreau K, Ludwig DS, et al. Prevalence of the metabolic syndrome in American adolescents: Findings from the third Nationa Health and Nutrition Examination Survey. Circulation 2004:110:2494-7.

[24] Alvarez MM, Vieira AC, Sichieri R, Veiga GV. Prevalence of metabolic syndrome and of its specific components among adolescents from Niteroi City, Rio de Janeiro State, Brazil. Arq Bras Endocrinol Metabol 2011;55:164-70.

[25] Sinaiko AR, Steinberger J, Moran A, et al. Relation of body mass index and insulin resistance to cardiovascular risk factors, inflammatory factors, and oxidative stress during adolescence. Circulation 2005;111:1985-91.
[26] Moraes AC, Fulaz CS, Netto-Oliveira ER, Reichert FF. [Prevalence of metabolic syndrome in adolescents: A systematic review]. Cad Saude Publica 2009;25:1195-202.

[27] Zimmet P, Alberti G, Kaufman F, et al. The metabolic syndrome in children and adolescents. Lancet 2007;369:2059-61.

[28] Cook S, Weitzman M, Auinger P, et al. Prevalence of a metabolic syndrome phenotype in adolescents: Findings from the third National Health and Nutrition Examination Survey, 1988-1994. Arch Pediatr Adolesc Med 2003; 157:821-7.

[29] Bedir A, Topbas M, Tanyeri F, et al. Leptin might be a regulator of serum uric acid concentrations in humans. Jpn Heart J 2003;44:527-36.

[30] Bosello O, Zamboni M. Visceral obesity and metabolic syndrome. Obes Rev 2000; $1: 47-56$

[31] Huang RC, Mori TA, Burke V, et al. Synergy between adiposity, insulin resistance, metabolic risk factors, and inflammation in adolescents. Diabetes Care 2009;32:695-701.

[32] Lemieux S, Despres JP. Metabolic complications of visceral obesity: Contribution to the aetiology of type 2 diabetes and implications for prevention and treatment. Diabete Metab 1994;20:375-93.

[33] Platis O, Anagnostopoulos G, Farmaki K et al. Glucose metabolism disorders improvement in patients with thalassaemia major after 24-36 months of intensive chelation therapy. Pediatr Endocrinol Rev 2004;2(Suppl 2): $279-81$.

[34] Reynisdottir S, Angelin B, Langin D, et al. Adipose tissue lipoprotein lipase and hormone-sensitive lipase. Contrasting findings in familial combined hyperlipidemia and insulin resistance syndrome. Arterioscler Thromb Vasc Biol 1997:17:2287-92.

[35] Stapleton FB, Linshaw MA, Hassanein K, Gruskin AB. Uric acid excretion in normal children. J Pediatr 1978;92:911-4.

[36] Jones DP, Richey PA, Alpert BS, Li R. Serum uric acid and ambulatory blood pressure in children with primary hypertension. Pediatr Res 2008;64: 556-61. 